\title{
Effects of media multitasking frequency on a novel volitional multitasking paradigm
}

\author{
Jesus J Lopez ${ }^{\text {Corresp., } 1}$, Joseph M Orr ${ }^{\text {Corresp. 1, } 2}$ \\ ${ }^{1}$ Department of Psychological and Brain Sciences, Texas A\&M University, College Station, Texas, United States \\ 2 Institute for Neuroscience, Texas A\&M University, College Station, Texas, United States \\ Corresponding Authors: Jesus J Lopez, Joseph M Orr \\ Email address: jesusjlopez619@tamu.edu, joseph.orr@tamu.edu
}

The effect of media multitasking (e.g., listening to podcasts while studying) on cognitive processes has seen mixed results thus far. To date, the tasks used in the literature to study this phenomenon have been classical paradigms primarily used to examine processes such as working memory. While perfectly valid on their own, these paradigms do not approximate a real-world volitional multitasking environment. To remedy this, as well as attempt to further validate previously found effects in the literature, we designed a novel experimental framework that mimics a desktop computer environment where a "popup" associated with a secondary task would occasionally appear. Participants could select the popup and perform a difficult word stem completion trial or ignore the popup and continue performing the primary task which consisted of math problems. We predicted that individuals who are more impulsive, more frequent media multitaskers, and individuals who prefer to multitask (quantified with the Barratt Impulsiveness Scale, a modified version of the Media Use Questionnaire, and the Multitasking Preference Inventory) would be more distracted by popups, choose to switch tasks more often and more quickly, and be slower to return to the primary task compared to those who media multitask to a lesser degree. We found that as individuals media multitask to a greater extent, they are slower to return to the previous (primary) task set and are slower to complete the primary task overall whether a popup was present or not, among other task performance measures. We found a similar pattern of effects within individuals who prefer to multitask. Our findings suggest that overall, more frequent media multitaskers show a marginal decrease in task performance, as do preferential multitaskers. Attentional impulsivity was not found to influence any task performance measures, but was positively related to a preference for multitasking. While our findings may lack generalizability due to the modifications to the Media Use Questionnaire, and this initial study is statically underpowered, this paradigm is a crucial first step in establishing a more ecologically valid method to study real-world multitasking. 
1

2 Effects of media multitasking frequency on a novel volitional multitasking paradigm

3

4

5 Jesus J Lopez ${ }^{1}$, Joseph M Orr',2

6

$7{ }^{1}$ Department of Psychological and Brain Sciences, Texas A\&M University, College

8 Station, Texas, United States

9

10

11

12

13

14

15515 Coke St., 4235 TAMU, College Station, Texas, 77843-4235, United States

16 Email address: joseph.orr@tamu.edu 


\section{Abstract}

The effect of media multitasking (e.g., listening to podcasts while studying) on cognitive processes has seen mixed results thus far. To date, the tasks used in the literature to study this phenomenon have been classical paradigms primarily used to examine processes such as working memory. While perfectly valid on their own, these paradigms do not approximate a realworld volitional multitasking environment. To remedy this, as well as attempt to further validate previously found effects in the literature, we designed a novel experimental framework that mimics a desktop computer environment where a "popup" associated with a secondary task would occasionally appear. Participants could select the popup and perform a difficult word stem completion trial or ignore the popup and continue performing the primary task which consisted of math problems. We predicted that individuals who are more impulsive, more frequent media multitaskers, or show a preference to multitask (quantified with the Barratt Impulsiveness Scale, the Media Use Questionnaire, and the Multitasking Preference Inventory, respectively) would be more distracted by popups, choose to switch tasks more often and more quickly, and be slower to return to the primary task compared to those who media multitask to a lesser degree. As predicted, we found that as individuals media multitask to a greater extent they are slower to return to the previous (primary) task set and were generally slower on primary task performance. We found a similar pattern of effects within individuals reporting a preference to multitask. Attentional impulsivity was not found to influence any task performance measures but was positively related to a preference for multitasking. While our findings may lack generalizability due to the modifications to the Media Use Questionnaire, and this initial study is statically underpowered, this paradigm is a crucial first step in establishing a more ecologically valid method to study real-world multitasking. 
42

43

44

45

46

47

48

49

50

51

52

53

\section{4}

55

56

57

58

59

60

61

62

63

64

65

66

67

68

69

70

71

72

73

74

75

76

77

78

79

80

81

82

83

\section{Introduction}

The preponderance of information available at our fingertips makes multitasking seem like the norm. Unsurprisingly, the proportion of time an individual multitasks with multiple information sources increased 10\% from 6 hours and 20 minutes a day, to 7 hours and 38 minutes a day between 1999 and 2009 (Rideout, Ulla, Foehr, \& Roberts, 2010). Furthermore, research suggests some negative impacts of screen time (i.e., time spent viewing television, phone/tablet, or laptop), on cognitive abilities and other psychosocial factors, and particularly on the development of these functions (Domingues-Montanari, 2017; Hooghe \& Oser, 2015; Sigman, 2012). As such, it is critical to understand the costs and potential benefits of frequent media multitasking, often defined as the simultaneous use of two or more media types or the act of quickly switching between different media types (Minear, Brasher, McCurdy, Lewis, \& Younggren, 2013).

\section{Previously found Effects of Media Multitasking}

To that end, research has aimed to establish differences in information processing as a function of time spent media multitasking, with a typical focus on extreme groups comparisons. A number of studies have now identified a negative association between media multitasking frequency and performance on cognitive tasks that require focus and cognitive stability such as distractor filtering (Lottridge et al., 2015; Moisala et al., 2016; Murphy \& Creux, 2021; Wiradhany \& Nieuwenstein, 2017), inhibitory control (Baumgartner, Weeda, van der Heijden, \& Huizinga, 2014; Schutten, Stokes, \& Arnell, 2017), and sustained attention (Ralph \& Smilek, 2017; Ralph, Thomson, Cheyne, \& Smilek, 2014). Thus far, frequent or heavy media multitasking exposure has been linked to deficits in single task settings, but research into domains where one might expect multitaskers to excel, such as task switching, has produced more mixed results. For example, Ophir and colleagues (2009) found a negative association between heavy media multitaskers and task switching, while Alzahabi and Becker (2013) found the opposite relationship. Indeed, a growing body of work suggests no relationship between media multitasking and task switching performance (Baumgartner et al., 2014; Minear, Brasher, McCurdy, Lewis, \& Younggren, 2013). More recently, Rogobete, Ionescu, and Miclea (2020) found that no linear relationship of media multitasking on task switching, but, when comparing extreme groups, the heavier media multitaskers counterintuitively performed better than low media multitaskers. Given these mixed results, more insight is necessary to describe the effect media multitasking has on this aspect of executive function.

The Media Use Questionnaire (MUQ) was developed by Ophir and colleagues (2009) to quantify the amount of time an individual media multitasks during a typical media-consumption hour. Participants are first asked how many hours a week they use different media sources, followed by how often they concurrently use each other media type. From this information it is possible to quantify an individual's Media Multitasking Index (MMI). Across a variety of executive function tasks, Ophir and colleagues found that heavy media multitaskers performed worse compared to those who multitask less. With this in mind, Ophir and colleagues suggested that heavy media multitaskers are less able to filter out irrelevant information when compared to 
84 their lighter media multitasking counterparts. In line with Ophir and colleagues, a number of

85

86

87

88

89

90

91

92

93

94

95

96

97

98

99

100

101

102

103

104

105

106

107

108

109

110

111

112

113

114

115

116

117

118

119

120

121

122

123

124

125 studies have now shown a similar pattern of results (Cain \& Mitroff, 2011; Cardoso-Leite et al., 2016; Heathcote et al., n.d.; Lottridge et al., 2015; Wiradhany \& Nieuwenstein, 2017).

Can propensity for media multitasking be predicted by individual emotional or attitudinal differences? Previous work indicates that sensation-seeking and impulsivity might influence the frequency of media multitasking. For example, research suggests that a weak positive association between total multitasking use and sensation seeking ratings on the Brief Sensation Seeking Scale exists (Jeong \& Fishbein, 2007). Similarly, sensation seeking has also been found to predict media multitasking frequency as measured by the MMI (Kononova, 2013). Sanbonmatsu and colleagues (2013) found that individuals with higher MMI scores tended to also score high on impulsivity, and moreover, performed worse on the Operation Span Task, a complex span task that involves rapid task switching, or multitasking, as defined by Madore and Wagner (2019). Furthermore, media multitasking has been found to be associated with attentional impulsivity, as measured by both performance on a Go/No-Go task and a subscale of the Barratt Impulsiveness Scale (BIS), as well as lower self-reported initiatory self-control (Shin, Webb, \& Kemps, 2019). Finally, Minear and colleagues (2013) found that heavy media multitaskers reported being more impulsive while also showing worse performance on measures of fluid intelligence. Taken together, these findings point towards the possibility of an emotional and cognitive basis behind this phenomenon. However, some research does suggest that the effects of screen time, at least in regard to adolescent well-being, have thus far been overstated and are in fact, much smaller than has been purported (Orben \& Przybylski, 2019).

Although most studies suggest a negative relationship between media multitasking and cognitive performance, a number have found no difference associated with media multitasking use, or even findings in the opposite direction. Indeed, a meta-analysis by Wiradhany and Nieuwenstein (2017) found a weak association between media multitasking and distractibility and a more recent meta-analysis by Parry and le Roux (2021) found a weak association between media multitasking and general cognitive function. These weak patterns of effects are prevalent in the task switching (Alzahabi \& Becker, 2013; Alzahabi, Becker, \& Hambrick, 2017; Schneider \& Chun, 2021), dual tasking, (le, Haller, Langer, \& Courvoisier, 2012), and inhibition literature (Rogobete et al., 2020). Interestingly, two studies have found that intermediate or moderate multitaskers show better $\mathrm{N}$-back performance compared to heavy and light media multitaskers (Cardoso-Leite et al., 2016; Shin, Linke, \& Kemps, 2020). Nevertheless, research examining intermediate or average media multitaskers is much less common than the extreme groups comparisons that the literature has to date focused on.

\section{Purpose}

In summary, there are still many outstanding questions regarding media multitasking's effect on task performance. Though the literature has found some effects, these have been derived from already established paradigms that have been historically used to study other cognitive processes that are not always immediately reminiscent of multitasking. In day-to-day life, multitasking is usually done at the leisure of the individual, with task switches occurring 
126

127

128

129

130

131

132

133

134

135

136

137

138

139

140

141

142

143

144

145

146

147

148

149

150

151

152

153

154

155

156

157

158

159

160

161

162

163

164

165

166

167

168

169

randomly and sporadically; this is counter to most lab-based studies of multitasking, in which the experimenter dictates when and how an individual multitasks. By giving participants the choice of when to switch to a secondary task, as well as modeling the task to be more similar to a multitasking environment, we can examine whether media multitasking frequency relates to one's tendency to switch tasks often as well as overall task performance. Thus, in the current study, we developed a novel experimental framework more analogous to multitasking in day-today life by having participants complete a primary, monotonous task with sporadic "interruptions" presented in the form of an opportunity to switch to a different, secondary task. We hope to use this paradigm to dispel the ambiguity in the current literature in the field by allowing us to more closely examine the differences between individuals' task performance and the effect extensive daily media multitasking may have on it by using a task specifically designed to emulate real-world multitasking. The ability to replicate previously established effects with this more ecologically valid paradigm would provide further support for those effects as well. Further, a majority of the literature has focused on an extreme groups approach. While this is obviously very valuable information to have, the question still remains as to whether any degree of media multitasking can affect task performance and not only in extreme "high" or "low" cases. The current work seeks to reconcile the limitations of the extreme groups approach, as well as establish a more ecologically valid task paradigm that can then be used to further examine cognitive differences and how they are affected by media multitasking.

In the current study, we operationalized the act of multitasking as the attempt to perform more than one task concurrently which then leads to the act of switching back and forth between tasks (Madore \& Wagner, 2019). To that end, we designed the framework of our novel paradigm around the Operation Span Task (OSPAN) devised by Turner and Engle (1989) as it requires participants to complete two tasks concurrently. In fact, Sanbonmatsu and colleagues previously used the OSPAN to examine multitasking ability (Sanbonmatsu et al., 2013). In our paradigm, a participants' primary task was a math problem verification task, similar to the OSPAN. However, in some trials, a pop-up message occasionally appeared which asked if the participant wanted to switch to a secondary task. The pop-up prompts were implemented to be reminiscent of the notifications that appear on our phones and computers and appeared randomly throughout a block of trials. If the participant indicated that they wanted to switch, they were then given a word stem completion to solve, after which they returned to the primary task. This is another differentiation from the OSPAN, as the secondary task in that paradigm is not optional and indexes an individual's working memory by asking participants to recall a series of letters that are presented after each primary task trial at the end of a block. In our current task, the participant does not have to hold any objects in their working memory as they work through the task, instead indexing their ability to task-switch.

Our paradigm also draws from the voluntary task switching (VTS) literature (Arrington \& Logan, 2015). We chose to model our paradigm from this literature because of the similar scenarios that are presented to participants in those paradigms. Here, the volitional aspect that is common in VTS tasks paradigms is present, albeit with some fundamental changes. The participant is only prompted to respond on a random subset of trials as opposed to having the option during each trial, a deviation from most VTS paradigms (Arrington \& Logan, 2004; Mayr \& Bell, 2006; Orr \& Weissman, 2011). For every trial in which the option to switch tasks is not presented, the participant is only able to complete the primary task. Again, this was done in an

Peer) reviewing PDF | (2021:04:60252:2:0:NEW 26 Oct 2021) 
170

171

172

173

174

175

176

177

178

179

180

181

182

183

184

185

186

187

188

189

190

191

192

193

194

195

196

197

198

199

200

201

202

203

204

205

206

207

attempt to further emulate a scenario in which real-world multitasking might occur. For example, an individual may be focused on a task on their computer, when a random popup in the corner of the screen may catch their eye. The individual then has the option to switch tasks away from their main focus to attend to this popup, a crucial element that is not present in VTS paradigms.

\section{Hypotheses}

We predicted a positive relationship between $\mathrm{MMI}$ score and the rate at which participants would elect to switch to the secondary task (Switch Rate). We also expected that participants would show a "Return Cost", i.e., respond slower to return to the primary task following a switch to the secondary task that would be positively predicted by media multitasking in line with the suggestion that media multitasking frequency is associated with decreased executive function (Baumgartner, Weeda, van der Heijden, \& Huizinga, 2014; Cain et al., 2016). Additionally, we predicted that individuals who media multitask more often would choose to switch to the secondary task more quickly (in the form of a faster time to elect to switch tasks on relevant trials, which we refer to as Popup $_{\text {select }}$ ), In line with the suggestion that frequent multitaskers show increased difficulties with distractor filtering, we predicted that MMI score would also show a positive relationship with the amount of interference exhibited on trials where a pop-up was presented, but the secondary task wasn't chosen (Interference Cost).

\section{Materials \& Methods}

\section{Participants}

A total of 90 participants (62 female, 28 male) with ages ranging from $18-23$ years $(M=$ $19.15, S D=0.9)$ fully completed the procedure. Two participants were dropped due to noncompletion of the study. Participants were recruited from the Texas A\&M Psychology Subject Pool and received course credit for participating. No target sample size was determined, with the intent to collect as much data as possible through the course of a full semester. A post-hoc power analysis was performed, described below. Demographic information is reported in Table 1. Participants were not prescreened for media multitasking frequency and only had to be English-speakers who were right-handed, neurotypical, had full color vision, and were between the ages of 18-30 years old; in addition, participants were not told this was a study on multitasking until they were consented to participate in the study. Study procedures were deemed exempt from the requirements of the Common Rule (45 CFR 46.101[b]) by the Texas A\&M Institutional Review Board, approval reference number IRB2018-1456M. The authors confirm that we have reported all measures, conditions, data exclusions, and the method of sample size determination.

\section{Media Multitasking Index}

A Media Multitasking Index (MMI) was calculated in order to assess the degree to which participants multitask with different forms of media (Ophir, Nass, \& Wagner, 2009). Participants 
208 first completed the Media Use Questionnaire, which asked participants to estimate how many

209 hours per week they use each individual form of media (using a sliding scale ranging from 0-80

210 (in hours). They were then given a matrix asking, for each media type they use, how often they

211 concurrently used each of the other mediums using a 5-point Likert scale ("Always," "Most of the

212 time," "Some of the time," "A little of the time," or "Never"). Although these values are not

213 disclosed to the participants, numeric values were assigned to each of the matrix answers, such

214 that "1.0" represented "Always", "0.75" corresponded to "Most of the time," "0.5" to "About half

215 the time", "0.25" to "Sometimes," and "0" to "Never." The sum of these values across primary

216 medium use weighted by the percentage of time spent with the corresponding primary medium

217 was then computed to yield a participant's Media Multitasking Index (MMI) score. This final MMI

218 score can be interpreted as the level of media multitasking the participant is engaged in during a

219 typical media-consumption hour so that the higher the MMI, the greater the amount of time that

220

221

222

223

224

225

226

227

228

229

230

231

232

233

234

235

236

237

238

239

240

241

242

243

244

245 participant spends media multitasking in an hour. Supplemental Figure S1 shows the equation Ophir and colleagues (2009) used for calculating MMI scores, again used in the current study. Briefly, the index is calculated by assigning numeric values to each of the matrix answers and weighing the sum of these values across each primary medium by the percentage of time spent with the corresponding primary medium.

The original version of the Media Use Questionnaire (Ophir et al., 2009) was modified for the current study to reflect current trends in media usage. This modified version assessed 12 media types; computer-based applications (e.g., word processing, excel), web surfing (not including social media sites), text-based media such as print books, ebooks, magazines, newspapers (for school/work/pleasure), television programs (TV based or online streaming), streaming videos (e.g., YouTube, BuzzFeed, other short clips), listening to music, listening to nonmusic audio (e.g., audio books, podcasts,, talk radio, etc.), video based games (console, computer, phone/tablet based), voice calls (landline, cellphone, skype), reading/writing emails, viewing social media (facebook, instagram, snapchat, twitter, etc.), and "other" media types. The original version of the questionnaire's "instant messaging" media type was replaced with "social media" to reflect the rise of social media and the decline of instant messaging since the creation of the questionnaire. We also changed the wording for several media types. "Print media" was changed to "text media" to reflect the popularity of e-readers, "telephone and mobile phone voice calls" was changed to "voice calls," "computer-based video" was renamed to "streaming video" (to reflect current trends towards services such as YouTube, Netflix, and Hulu), and "video or computer games" was renamed to "video games." Ophir et al.'s version of the index used only a 4-point Likert scale ranging from "Most of the time" to "Never". We added the additional answer choice of "Always" in an attempt to get a more precise measure of media multitasking occurrence. The addition of the extra choice of "Always" was done to counterbalance the already existing "Never" answer choice.

\section{Multitasking Preference Inventory (MPI)}

Participants also completed the Multitasking Preference Inventory, a 14-item questionnaire devised by Poposki and Oswald (2010) to index an individual's general "preference towards multitasking." It consists of fourteen statements relating to their opinions on 
250

251

252

253

254

255

256

257

258

259

260

261

262

263

264

265

266

267

268

269

270

271

272

273

274

275

276

277

278

279

280

281

282

283

284

285

286

287

288

289

performing tasks (ex: "I prefer to work on several projects in a day, rather than completing one project and then switching to another.") that they then indicate on a 1 (Strongly Disagree) to 5 (Strongly Agree) Likert scale as to how well each describes them. Scoring was done in accordance with Poposki and Oswald (2010) and includes the summation of all items once the appropriate questions have been reverse scored. Higher scores on this measure suggest a higher inclination to want to multitask.

\section{Barratt Impulsiveness Scale-11 (BIS-11)}

In order to assess impulsivity, the BIS-11 was administered and scored according to previous works, consisting of the sum of all items following the reverse scoring of the appropriate questions. (Patton, Stanford, \& Barratt, 1995). The questionnaire consists of 30 items on a 1 (Rarely/Never) to 4 (Almost A/ways/A/ways) Likert scale related to impulsive behaviors and attitudes. The scale can be further broken down into 6 first order factors (Attention, Cognitive Instability, Motor, Perseverance, Self-Control, and Cognitive Complexity) and 3 second order factors (Attentional, Motor, and Nonplanning). Following Sanbonmatsu et al. (2013), all 30 questions of the BIS-11 were used, with the Attentional impulsivity sub-scale being especially of interest for the current study

\section{Multitasking Paradigm}

Figure 1 is a representation of the multitasking paradigm. Participants completed a computerized multitasking paradigm, created using PsychoPy version 3.0.6 (Peirce et al., 2019), on a 21-inch iMac (Apple, Inc.). All monitor settings were determined by the default test monitor settings within PsychoPy. For the primary task, participants checked the validity of math operations (e.g., '( $3-2) \times 1=4$ ') via key press, with " $C$ " indicating the math problem was correct, and "l" to indicate an incorrect problem. The math operations were on the screen for 5 seconds. Participants were informed that a correct response to the primary task was worth 3 points. Incorrect or responses not made in time would deduct this same amount from the total. Participants were shown their running total after every trial. The points did not have a monetary value, but to incentivize participants to achieve as high a score as possible, they were shown a "high score" at the end of each block. This high score was the same for each participant.

On one out of every six primary task trials, a text "popup" would appear on the screen $500 \mathrm{~ms}$ after the primary task appeared, reading "A New Task is Available! Press ' $Y$ ' to switch tasks". This popup would appear on the top right corner of the screen. The position of the popups was chosen so that they would be reminiscent of the notifications seen on computers and cell phones. The popup would appear on screen for 2 seconds, after which the text would disappear. Participants could choose to continue attending to the primary task instead of the popup. If participants chose to switch tasks, they would then be shown a word fragment with two letters missing (e.g., "HI_TO_Y"). Participants would then indicate which letters were missing via 
290

291

292

293

294

295

296

297

298

299

300

301

302

303

304

305

306

307

308

309

310

311

312

313

314

315

316

317

318

319

320

321

322

323

324

325

326

327

328

329

key press. A correct response to the secondary task was worth 10 points, with an incorrect or response not made in time would deduct this same amount from the total. The discrepancy of possible points between the primary and secondary tasks was implemented to make the secondary task more enticing and encourage multitasking, due to the greater amount of points possible for successfully completing it. Participants again were shown their running total at the end of each trial.

The task consisted of 8 blocks of 20 trials each for a total of 160 trials. The number of blocks was chosen so that the task would be broken up into intervals allowing the participant to take breaks regularly while still being able to complete the experiment in under an hour. The number of pop-ups was not consistent across participants due to the randomization procedure, with an average of $27.8(S D=4.4)$ pop-ups per participant.

\section{Procedure}

After providing consent via a written consent form, participants completed an online version of the Media Use Questionnaire, the MPI, the BIS-11, and a demographics questionnaire. After completion of the surveys, participants then completed a short practice version of the novel multitasking paradigm, followed by the full version of the task. The practice version of the task consisted of three distinct blocks. In the first block, participants completed 5 trials of only the primary task. Similarly, they completed 5 trials of only the secondary task in the second block. In the final practice block, participants completed 6 trials of the full task. Point values were identical to the full task. The total experiment duration was about 1 hour.

\section{Data Analysis}

Analyses and plots were created using RStudio Version 1.2.5033 (R Core Team, 2019). Post hoc power analysis was conducted using G*Power (Faul, Erdfelder, Buchner, \& Lang, 2009). Survey scores were compared using Pearson's 2-tailed correlations. The main dependent measures for task performance were switch rate (the percentage of trials in which a participant switched tasks across all trials in which switches were possible), Popup select $_{\text {, }}$ or the reaction time for individuals to elect to switch tasks on relevant trials, Return Cost, i.e., the difference in average reaction time for primary tasks following a switch to the secondary task minus the average reaction time for all other primary task trials without a popup, and Interference Cost, i.e., the difference in reaction time for primary task trials with a non-selected pop-up and primary task trials without a pop-up.

Because some participants did not switch at all throughout the task $(n=23)$, it was not possible to calculate some measures for the entire sample. The effects of media multitasking on task performance were analyzed using a hierarchical regression model consisting of the three main surveys (Attentional BIS, MMI, and MPI) to predict each measure of task performance. In the first step of the regression, we included only MMI Score, as that was the main construct of interest. In step 2, we then included the attentional sub-scale of the BIS score, with MPI score being added in step 3 . 
330

331

332

333

334

335

336

337

338

339

340

341

342

343

344

345

346

347

348

349

350

351

352

353

354

355

356

357

358

359

360

361

362

363

364

365

366

367

368

369

To maximize the amount of useable data, all trials in which a participant responded to either task were included in our analyses, unless otherwise noted. The data and materials for this experiment are available at

(https://osf.io/nju8a/?view_only=27e3adfafbba48488a1bf0f7c20e1f4a). This experiment was not preregistered.

\section{Results}

\section{Survey Results}

Table 1 shows a breakdown of survey scores. Mean MMI was 2.95 (SD =1.3), with significant deviation from normality $(W=0.96, p=.01)$. There was no difference in MMI between males and females, $F(1,88)=1.77, p=.19, \eta_{p}^{2}=0.02$. Our mean MMI is relatively in line with that of other studies using the original MUQ questionnaire and its method of calculation devised by Ophir and colleagues (2009) (Moisala et al., 2016; Ralph et al., 2014; Schneider \& Chun, 2021).

The mean MPI score was $38.5(S D=10.9)$, indicating an overall neutral preference for multitasking. This is slightly higher and less variable than previous studies that have also used this measure, suggesting that our sample had a slightly greater preference for multitasking. For example, a random sample of experienced Amazon MTurk workers resulted in an average of 38.01 ( $S D=12.54$ ) (Lascau, Gould, Cox, Karmannaya, \& Brumby, 2019). Relatedly, an inperson sample of university students found an average MPI score of 29.95 ( $S D=8.72$ ) (Magen, 2017).

Median Total BIS was $61.0(S D=9.7)$, with a median Attentional score of $17.0(S D=$ $3.9)$, a median Motor score of $20.0(S D=4.3)$, and a median Nonplanning score of $23.5(S D=$ 4.2). MMI was significantly correlated with BIS-Motor $(r=0.27)$, and MPI scores were correlated with Total BIS $(r=0.26)$ as well as BIS-Attentional $(r=0.29)$, BIS-Cognitive Instability $(r=.23)$, BIS-Self Control $(r=.23)$ and BIS-Motor $(r=0.22)$ sub-scale scores. However, MMI was not correlated with MPI $(r=-0.1)$. Expectedly, all of the BIS sub-scales were correlated with Total BIS (all $r>0.74$ ). Supplemental Table S1 shows a correlation matrix of all surveys and behavioral measures.

\section{Multitasking Performance}

Participants performed the primary task (math problem verification) with high accuracy $(\mathrm{M}=94.3 \%, \mathrm{Cl}=91.8-97.1 \%)$ and the secondary task (word stem completion) with moderate accuracy $(M=69.7 \%, C l=59.1-89.2 \%)$. Popups appeared on a median of 28 trials $(C l=24.25-$ 30.75 ), and participants chose to switch to the secondary task on an average of $30.8 \%$ of popup trials $(C l=22.6-54.7 \%)$. According to a Wilcoxon one sample t-test, this value significantly differed from $0.0(V=2278, p<.001, r=1.0)$, however, 23 participants did not respond to any of the popups, with an additional 8 only responding to 1 . Primary task reaction time was then 
370

371

372

373

374

375

376

377

378

379

380

381

382

383

384

385

386

387

388

389

390

391

392

393

394

395

396

397

398

399

400

401

402

403

404

405

406

407

408

409

410

411

412

413

analyzed in a repeated measures ANOVA with one factor with the following levels: Ignore (i.e., popup was present but not responded to), Return (i.e., previous trial on which the secondary task was performed), and No Popup (i.e., no popup on current or previous trial). Only correct trials were included in this analysis and participants with less than 3 values in any cell were excluded, resulting in a final sample of 50 participants. There was a main effect of condition $\left(F(1.32,64.7)=24.9, p<0.01, \eta_{g}^{2}=0.08\right)$, and pairwise tests showed significant differences between No Popup and Return (i.e., Return Cost), Return and Ignore, but not No Popup and Ignore (i.e., Interference Cost), as shown in Figure 2. A similar analysis was run for accuracy data (as in transformed), and no effect of condition was observed $(F(1.71,85.72)=1.4, p=$ $\left.0.25, \eta_{g}^{2}=0.017\right)$.

Next, we examined whether multitasking behavior was predicted by the multitasking and personality surveys. Table 2 shows a breakdown of the main behavioral measures analyzed

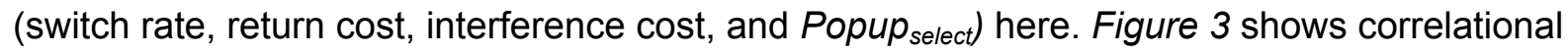
plots between the main behavioral measures analyzed and MMI score. Because we took a hierarchical regression modeling approach, we conducted three separate post hoc power analyses on the main analyses described using $G^{*}$ power (Faul et al.,2009), one for each separate model added.

Switch rate. The hierarchical model predicted switch rate (the percentage of trials in which a participant switched tasks across all trials in which a popup occurred) only in step 3 (Full model: $F(3,86)=5.6, p=.001$, Adjusted $R^{2}=.13$ ). Only step 3 of the model achieved greater than $80 \%$ power according to a post-hoc power analysis. Individual predictors in the model were examined further, and only MPI score predicted switch rate $(B=0.01, S E=0.003, t=3.8, p=<.001)$. Given that the MPI is thought to reflect the tendency or preference to multitask, we expected MPI to relate to switch rate, which was supported by our results. Supplemental Table S2 shows the hierarchical model at each step for this variable. To more directly relate MPI and switch rate, we correlated non-zero switch rate and MPI scores, and found a significant positive correlation $(r(65)=.33, p=.007)$, suggesting that the tendency to multitask in day-to-day life, as indexed by the MPI, does indeed have at least a weak association with participants' choice to switch to the secondary task when given the opportunity.

Return cost. None of the steps in the hierarchical regression model were significant for return cost, or the difference in average reaction time for primary tasks following a switch to the secondary task minus the average reaction time for all other primary task trials without a popup (Full model: $F(3,61)=1.42, p=.25$, Adjusted $R^{2}=.02$ ). A post hoc power analysis also suggested that we did not reach the sample size necessary to achieve above $80 \%$ power on any of the three steps of the model. We expected return cost to be related to MMI score, in line with previous work suggesting that media multitaskers show a decrease in task performance, but this was not the case. Supplemental Table S3 shows the hierarchical model at each step for this variable.

Interference cost. The hierarchical model predicting interference cost, or the difference in average reaction time for primary task trials with a non-selected pop-up and average reaction 
414 time on primary task trials without a pop-up was not significant at any step (Full model: $F(3,85)$ $415=0.69, p=.56$. Adjusted $\left.R^{2}=-.01\right)$. A post hoc power analysis also suggested that we did not 416 reach the sample size necessary to achieve above $80 \%$ power on any of the three steps of the 417 model. Supplemental Table S4 shows the hierarchical model at each step for interference cost.

418

419

420

421

422

423

424

425

426

427

428

429

430

431

432

433

434

435

436

437

438

439

440

441

442

443

444

445

446

447

448

449

450

451

452

453

454

455

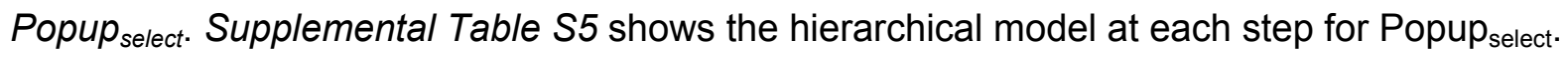
None of the three models predicting Popup select, or the RT for participants to choose to switch after popup onset on relevant trials, were significant (Full model: $F(3,63)=0.93, p=.43$, Adjusted $\left.R^{2}<.001\right)$. A post hoc power analysis also suggested that we did not reach the sample size necessary to achieve above $80 \%$ power on any of the three steps of the model. Our pattern of results here suggests that there was no difference in the amount of time an individual took to elect to switch tasks in relevant trials in terms of degree of multitasking, impulsivity score, or preference for multitasking.

\section{Exploratory Analyses}

As this is a novel task with many components, we made several exploratory comparisons to examine the relationships between $\mathrm{MMI}$ and task performance. To this end we examined RT on trials in which the participant ignored the popup and completed the primary

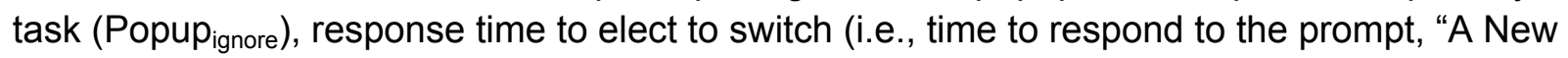

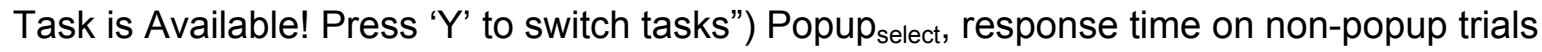
(Primary nopopup), RT on primary trials following a switch, regardless of availability of a switch

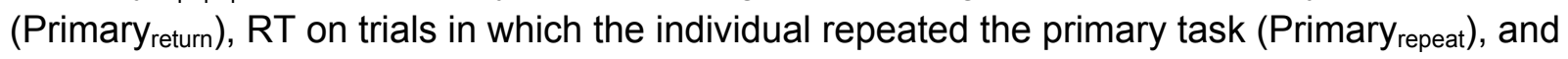
overall average RT on the primary and secondary tasks were also determined for each participant. As before, because some participants did not switch tasks at all, some of these measures could not be calculated for the entire sample. We again used a hierarchical multiple regression analysis to develop a model predicting each of these measures based on survey results. The first step of the model added MMI score to the model, while steps 2 and 3 added attentional impulsivity scores and MPI scores, respectively. Supplemental Table S6 shows a breakdown of the exploratory analyses described here. Additionally, we compared individuals who did not switch at all during the task to those who did on each task and survey measure (where possible) using both parametric and non-parametric t- tests (where appropriate, as some task measures were non-normally distributed) to examine for any differences in task performance between both groups. Figure 4 shows correlational plots between the exploratory behavioral measures analyzed and MMI score. We again note that the current study did not achieve the sufficient statistical power needed to detect the weak effect of media multitasking on task performance, which may explain the pattern of effects found.

Survey Results. A Wilcoxon Signed-Ranks test suggested that there was no difference in MMI scores between those who did not switch at all throughout the task (median $=2.52$ ) and those who did(median $=2.87$ ) $W=847, p=.76$. There was a significant effect for switch group, $t(59.9)$ $=-3.29, p=.001$, indicating that those who did not switch at all had a lower MPI score $(M=33.4$, $S D=8.17)$ than those who did switch $(M=40.5, S D=11.27)$. A Wilcoxon Signed-Ranks test 
456

457

458

459

460

461

462

463

464

465

466

467

468

469

470

471

472

473

474

475

476

477

478

479

480

481

482

483

484

485

486

487

488

489

490

491

492

493

494

495

496

497

498

499

suggested that there was an effect of switch group on the attentional impulsivity sub-scale of the BIS, $W=562.5, p=.02$, between those who did not switch at all throughout the task (median = 32 ) and those who did(median $=38$ ). These results suggest that both attentional impulsivity and preference for multitasking are positively related to the act of switching throughout the task in the current study.

Primary $_{\text {return. }}$ Supplemental Table S7 shows the hierarchical model at each step for this

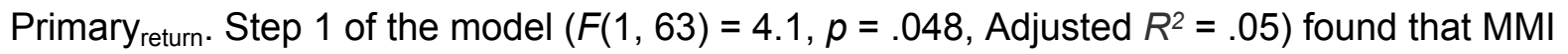
score predicted Primary return $(B=0.15, S E=0.072, t=2.02, p=.48)$. Steps 2 and 3 were not significant (Full model: $F(3,61)=1.47, p=.23$, Adjusted $R^{2}=.02$ ). Our finding in step 1 of the model suggests that those who media multitask more often show a decrease in their ability to return to an initial task following a switch in task set, such that they are slower to respond to the initial task regardless of the availability of a switch on that given trial. The lack of a relationship

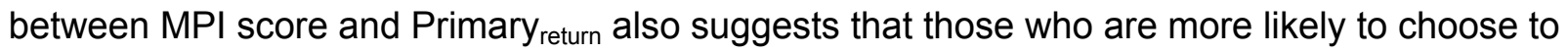
multitask do not show an increase in performance when switching back and forth between task sets.

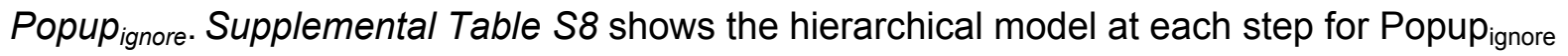
i.e., the reaction time on trials in which a popup occurred but was not attended to. The initial

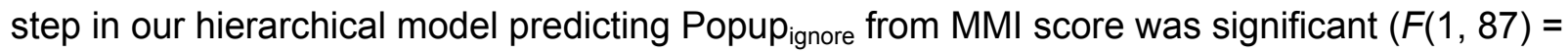
$\left.6.08, p=.02, R^{2}=.05\right)$. MMI score positively predicted popup interference in the form of a longer $\mathrm{RT},(B=0.09, \mathrm{SE}=0.037, t=2.47, p=.02)$. Step 2 in the model was also significant $\left(F(2,86)=3.15, p=.048\right.$, Adjusted $\left.R^{2}=.05\right)$, but the change in $R^{2}$ was not. The step 3 model was also significant $\left(F(3,85)=4.28, p=.007\right.$, Adjusted $\left.R^{2}=.1\right)$, as was the change in $R^{2}$. A relationship between MMI score and Popup ignore $\mathrm{RT}$ suggests that the more an individual media multitasks, the slower they are on trials in which they decide to ignore popups, in line with the suggestion that heavy media multitaskers have difficulty filtering irrelevant information (Cain \& Mitroff, 2011; Ophir et al., 2009). The relationship between MPI score and Popup ignore $_{\text {suggests }}$ that although individuals may have a preference for multitasking, they may still be unable to filter out irrelevant information during a task. There was no significant effect for switch group, $t(56.6)$ $=-1.395, p=.018$, despite those who switched during the $\operatorname{task}(M=2.36, S D=0.48)$ having a longer RT than those who did not switch at all $(M=2.23, S D=0.37)$ on trials in which a popup occurred but was ignored.

Primary nopopup. Supplemental Table S9 shows the hierarchical model at each step for this variable. The initial model predicting response time on tasks in which there was no popup

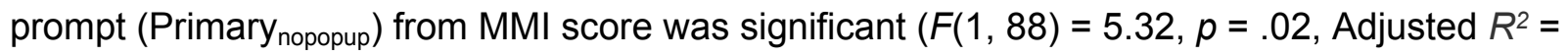
.05). MMI score positively predicted RT on primary task trials with no popup prompt $(B=.08, S E$ $=0.034, t=2.31, p=.02)$. Step 2 of the hierarchical model was not significant, but step 3 was (Full model: $F(3,86)=3.34, p=.02$ Adjusted $R^{2}=.07$ ). In this model, MPI score positively predicted RT on primary task trials with no popup prompt $(B=.008, S E=0.004, t=1.99, p=$ $.05)$, as did MMI score ( $B=.08 \mathrm{SE}=0.03, t=2.5, p=.01)$. The relationship found between MMI score and Primary nopopup suggests that individuals who media multitask more often are generally slowed, while the relationship between MPI score and Primary nopopup $_{\text {suggests that preferential }}$ 
500 multitaskers show this same pattern of effects. A Wilcoxon Signed-Ranks test suggested that

501

502

503

504

505

506

507

508

509

510

511

512

513

514

515

516

517

518

519

520

521

522

523

524

525

526

527

528

529

530

531

532

533

534

535

536

537

538

539

540

541 there was no difference in RT scores on trials in which no popup occurred between those who did not switch at all throughout the task (median $=2.18$ ) and those who did(median $=2.33) W=$ $660, p=.17$.

Primary $R T$. The initial step in the model predicting response time on all primary trials from MMI score was significant $\left(F(1,88)=5.16, p=.03\right.$, Adjusted $\left.R^{2}=.04\right)$. MMI score positively predicted Primary $\mathrm{RT}(B=0.08, \mathrm{SE}=0.034, t=2.27, p=.08)$. Step 2 was not significant, but step 3 was, $\left(F(3,86)=3.41, p=.02\right.$, Adjusted $\left.R^{2}=.08\right)$. The individual predictors in the model were examined further, and both MPI score $(B=0.01, S E=0.004, t=2.08, p=.04)$ and $\mathrm{MMI}$ score $(B=0.08, S E=0.03, t=2.49, p=.01)$ positively predicted Primary RT. Higher MMI and MPI was associated with slower RT on the primary task, suggesting an overall slowing for heavier media multitaskers, as well as those who prefer to multitask in general. Supplemental Table S10 shows the hierarchical model at each step for this variable. A Wilcoxon SignedRanks test suggested that there was no difference in RT scores on trials in which the participant completed the primary task on the preceding trial between those who did not switch at all throughout the task (median $=2.2$ ) and those who did(median $=2.33), W=664, p=.18$.

Secondary RT. All three steps of the models predicting RT on the secondary task were not significant (Full model: $F(3,60)=0.26, p=.85$, Adjusted $R^{2}=<.001$ ). Supplemental Table S11 shows the hierarchical model at each step for secondary $R T$.

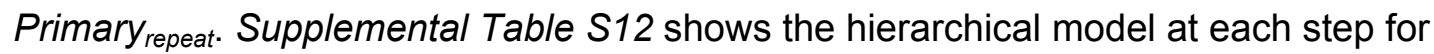

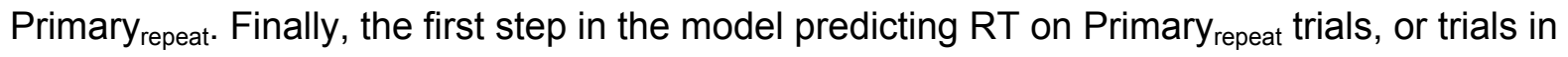
which the participant completed the primary task on the preceding trial, from MMI score was significant $\left(F(1,88)=4.69, p=.03\right.$, Adjusted $\left.R^{2}=.04\right)$. MMl score positively predicted $(B=$ $0.07, S E=0.034, t=2.17, p=.03$ ) reaction times on trials in which the primary task was also completed on the preceding trial. Step 2 was not significant, but the final step, which added MPI score to the hierarchical model, was (Full model: $\mathrm{F}(3,86)=3.1, \mathrm{p}=.03$, Adjusted $R^{2}=.07$ ). MPI score positively predicted $(B=0.008, S E=0.004, t=2.04, p=.04)$ reaction times on trials in which the primary task was also completed on the preceding trial, as did MMI score $(B=0.08$, $S E=0.03, t=2.39, p=.02)$. This pattern of results suggests that those who media multitask as well as prefer to multitask more often are slower when attending to the same task for a prolonged period of time.

A Wilcoxon Signed-Ranks test suggested that there was no difference in RT scores on trials in which the participant completed the primary task on the preceding trial between those who did not switch at all throughout the task (median $=2.21)$ and those who $\operatorname{did}($ median $=2.28) W=$ $698, p=.30$.

\section{Discussion}

In this study we investigated the effects of self-reported media multitasking exposure on performance in a novel multitasking paradigm. This paradigm consisted of a volitional task 
542 switch prompted by random text popups during the primary task; critically, participants were

543 able to ignore or choose to engage with the popup. If they chose to engage with it, they would

544 then complete a different secondary task before returning to the primary task. Participants were

545 not pre-selected for extreme degrees of media multitasking as in many previous studies; we

546 took an individual difference approach using naïve participants. We used hierarchical regression

547 models to predict task performance based on self-reported media multitasking exposure and

548 preferences and the Attentional impulsivity subscale of the BIS-11.

549

550

551

552

553

554

555

556

557

558

559

560

561

562

563

564

565

566

567

568

569

570

571

572

573

574

575

576

577

578

579

580

581

582

583

584

585
We hypothesized that media multitasking exposure (MMI score) and preference (MPI score) would predict both the frequency at which participants would elect to switch to the secondary task (switch rate), as well as the RT to choose to switch on relevant trials

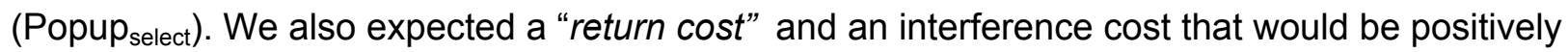
predicted by media multitasking scores. In addition to these initial constructs of interest, we also performed several exploratory analyses between the survey constructs and several other behavioral measures. These included the effect of each survey measure on the RT on primary task responses following a task switch (Primary ${ }_{\text {return }}$ ), primary task RT on trials where a pop-up was presented, but the secondary task wasn't chosen (Popup ignore), RT on trials in which no popup was present (Primary nopopup $_{\text {) }}$ RT on primary task trials in which the participant completed

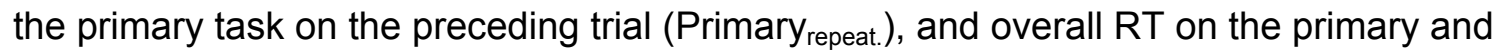
secondary tasks.

We found mixed results. In line with our primary hypotheses, we found that MPI score predicted switch rate. However, we found no significant predictors of return cost. Several exploratory analyses yields results supporting the hypothesis that media multitasking exposure relates to poorer executive function; we found that MMI score positively predicted Primary return, Popup $_{\text {ignore }}$, Primary $_{\text {nopopup }}$, Primary $_{\text {repeat, }}$ and primary RT. We also found that MPI score positively

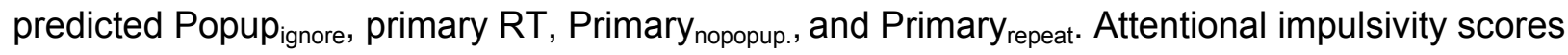
on the BIS-11 subscale were not substantial, with the exception of when comparing results across individuals who did not switch at all versus those who did. After comparing the survey scores of individuals who did not switch at all throughout the task versus those who did, we also found that those who switched tasks had higher MPI and attentional impulsivity scores than those who did not switch tasks at all. Although we found several significant models and predictors, it is crucial to underline the fact that the effect sizes for all findings were small and as such are likely not indicative of any greater underlying trend. In fact, according to a post-hoc power analysis using $\mathrm{G}^{*}$ power (Faul et al., 2009), the current study did not achieve the sufficient statistical power needed to detect the weak effect of media multitasking on task performance.

Supporting the idea that high media multitaskers show less efficient executive functioning (Becker, Alzahabi, \& Hopwood, 2013; Cain \& Mitroff, 2011; Murphy \& Creux, 2021; Ophir et al., 2009; Sanbonmatsu, Strayer, Medeiros-Ward, \& Watson, 2013), we found that greater were an individual's MMI and MPI scores, the greater was their RT on primary trials following a switch, regardless of a popup being present. This suggests that media multitasking reduces one's ability to re-engage with the primary task. This has been demonstrated in applied domains such as multitasking while driving (Nijboer, Borst, van Rijn, Taatgen, 2016; Strayer, 
586

587

588

589

590

591

592

593

594

595

596

597

598

599

600

601

602

603

604

605

606

607

608

609

610

611

612

613

614

615

616

617

618

619

620

621

622

623

624

625

626

627

628

629
Watson, Drews, 2011). However, our results regarding return cost, or the difference in RT on primary trials with no popup available following a task switch, may contest this interpretation. In regard to return cost, we found no effects within the three steps of our model. This may suggest that although heavier media multitaskers are less effective when switching back to a task from a previous task set on average, this difference is not detectable when only taking into account primary task RT on trials following a switch in which another switch is not possible. Additionally, both MMI and MPI score predicted overall RT on all primary task trials. Here, those who media multitask more often, as well as those who prefer to multitask, responded to the primary task more slowly in general. These results point toward a general decrease in task performance for individuals who engage in media multitasking more often.

Heavy media multitaskers have been found to have an inability to efficiently filter out distractors (Lui \& Wong, 2012; Murphy \& Creux, 2021; Ophir et al., 2009). Our results suggest a similar relationship, with individual MMI score predicting reaction time during Popup ignore trials, or trials in which a popup occurred but the participant chose not to switch, such that responses to the primary task during these trials were slower for individuals who media multitask more often. In trials in which the participant chose not to switch, the popup can be seen as a distraction from completing the primary task. As such, a longer RT to complete the primary task here demonstrates an inability to effectively filter irrelevant stimuli to the task at hand. This pattern of effects is true regarding MPI score as well, suggesting that even preferential multitaskers may be distracted to a greater extent by a popup stimulus, even if they choose to ignore the option to switch tasks. However, our findings regarding interference cost, or the difference in RT on trials in which the participant ignored a popup and the RT on trials in which no popup occurred, may conflict with this interpretation. The lack of a relationship here may be attributed to the low amount of overall switches, which are further elaborated on below. The weak effect size associated with the former finding may also account for this discrepancy.

Because greater impulsivity and worse inhibitory control have been linked to MMI scores (Gorman \& Green, 2016; Sanbonmatsu et al., 2013; Murphy \& Creux, 2021; Rogobete et al., 2021; Shin et al., 2019), we expected a greater switch rate among more impulsive and less inhibited individuals. However, we found no evidence that attentional impulsivity as indexed by the sub-scale of the BIS score predicts switch rate. We did, however, find that individuals who did not switch tasks at all had lower attentional impulsivity scores than those who did, along with a lower preference for multitasking. We found similar results for the RT for individuals to choose

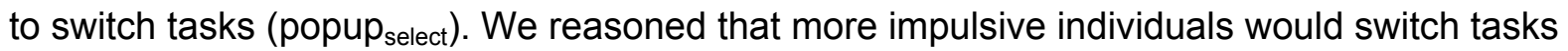
more quickly and frequently, again because of the greater possibility of reward due to completing more the secondary task.

Several factors may have contributed to the low switch rate observed in the current study $(\sim 31 \%)$. For example, the popup prompts may not have been salient enough to entice a switch. Increasing the points earned for completing the secondary task or making the popup more prominent on the screen by changing the text color or including sound may make the popups more salient. Because there was no monetary incentive for a higher score other than the motivation to "beat" a "high score", participants may have had no motivation to maximize 
630 points earned, leading to less task switches. This is a limitation of the task we must

631 acknowledge, as we have no way to be certain that this did not affect our participants'

632 motivation and thus, our results.

633

634

635

636

637

638

639

640

641

642

643

644

645

646

647

Relatedly, the greater penalties for an error in the secondary task may have also disincentivized task switches. Interestingly, higher MPI score was related to switch rate, but MMI score was not. Individuals who switched tasks had higher MPI scores when compared to those who did not switch at all as well. These effects point towards a greater propensity for preferential multitaskers to opt to switch to a different task set, but not for individuals who report engaging in media multitasking to a greater degree. Finally, there was a non-trivial difference in overall participant accuracy between the primary task (93\%) and the secondary task (58\%); one possibility is that participants found the secondary task too difficult and not worth the increased effort (Inzlicht, Shenhav, Olivola, 2018). This was not analyzed further due to the even greater potential for incomparable data due to the overall low switch rate observed.

The changes we made to the Media Use Questionnaire may have also contributed to some of the findings, both null and significant, in this experiment. Many of the changes made to the original 2009 questionnaire devised by Ophir and colleagues were done to reflect changes

648

649 in the media consumption landscape we see today. Nevertheless, our average MMI score was relatively in line with other studies that have used the original Ophir (2009) questionnaire.

650

651

652 Despite this, we must still acknowledge that the changes made in the current study to the original questionnaire may limit the generalizability of our findings to other studies that used the original version. Since the original introduction of the media use questionnaire in 2009 , there

653

654

655 have been attempts to devise a more cohesive and brief version of the questionnaire, with differing patterns of effects (Baumgartner et al., 2016; Pea, Nass, Meheula,, Rance, Kumar, Bamford, Nass, Simha, Stillerman, Yang, Zhou, 2012.). This lack of uniformity in regard to

656

657 screen time and media use measures in the overall literature points to a bigger problem recently emphasized by Kaye and colleagues (2020). They point out that the conceptualization of media use is far too broad and ambiguous in its current state in the literature, and vastly undermines

659

660

661 the generalizability of the literature to a broader audience.

662

Despite the limitations discussed, the findings resulting from this novel multitasking paradigm are promising. Because the majority of current media multitasking literature has used paradigms designed to evaluate other domains of executive function such as working memory and inhibitory control, the implementation of a paradigm specifically designed to be analogous to the environment in which individuals frequently multitask is needed. This initial study serves as a first step to fill this gap in the literature. Further implementations to this paradigm to develop a task more analogous to real world multitasking should include a sound clip in conjunction with the popup notification. This would be reminiscent of many of the notifications we receive on our phones and laptops, as they too may sometimes include sound. Many of the notifications we receive on these same devices can be ignored as they are "spam" or of little interest to us. As such, the inclusion of uninformative or "distractor" popups mixed in with informative popups may serve to further emulate a real-world multitasking environment. It may be beneficial to include trials in the paradigm where a task switch is required to allow for a clear 
674 differentiation between an interference cost and trials in which a participant actively chooses to 675 switch costs, as in our current design, this is not possible. Finally, a larger sample size is 676 needed to provide for enough statistical power to detect effects our paradigm may uncover.

677

\section{Conclusions}

679

680

681

682

683

684

685

686

687

688

689

690

691

692

693

Using a novel, more ecologically valid paradigm, we expected to find a negative effect of media multitasking, multitasking preference, and attentional impulsivity on task performance. We found a number of significant, albeit weak, effects of media multitasking on task performance, including a general slowing effect on the primary task. We also found that selfreported multitasking preference related to how often participants chose to engage in the secondary task. These findings contribute to the now growing media multitasking literature showing some of the negative effects of frequentmedia multitasking. However, it is crucial to recognize that many of the effects we found were weak, and with a smaller than ideal sample size, may not persist given further testing. Further, the adjustments made to the Media Use Questionnaire use may limit the generalizability of our findings to the work done using the questionnaire in its original form. Future directions of this line of research include a modification to the paradigm to make the popup prompts more "enticing" to participants to more closely mirror a real-world multitasking environment. We also plan to collect EEG recordings to examine 694 the event related potentials occurring as participants complete the tasks.

695 
696

697

698

699

700

701

702

703

704

705

706

707

708

709

710

711

712

713

714

715

716

717

718

719

720

721

722

723

724

725

726

727

728

729

730

731

732

733

734

735

736

737

738

\section{References}

Alzahabi, R., \& Becker, M. W. (2013). The association between media multitasking, taskswitching, and dual-task performance. Journal of Experimental Psychology: Human Perception and Performance, 39(5), 1485-1495. https://doi.org/10.1037/a0031208

Alzahabi, R., Becker, M. W., \& Hambrick, D. Z. (2017). Investigating the relationship between media multitasking and processes involved in task-switching. Journal of Experimental Psychology: Human Perception and Performance, 43(11), 1872-1894. https://doi.org/10.1037/xhp0000412

Arrington, C. M., \& Logan, G. D. (2004). The Cost of a Voluntary Task Switch. Psychological Science, 15(9), 610-615. https://doi.org/10.1111/j.0956-7976.2004.00728.x

Baumgartner, S. E., Lemmens, J. S., Weeda, W. D., \& Huizinga, M. (2016). Measuring Media Multitasking. Https://Doi.Org/10.1027/1864-1105/A000167. https://doi.org/10.1027/18641105/A000167

Baumgartner, S. E., Weeda, W. D., van der Heijden, L. L., \& Huizinga, M. (2014). The Relationship Between Media Multitasking and Executive Function in Early Adolescents. The Journal of Early Adolescence, 34(8), 1120-1144. https://doi.org/10.1177/0272431614523133

Becker, M. W., Alzahabi, R., \& Hopwood, C. J. (2013). Media Multitasking Is Associated with Symptoms of Depression and Social Anxiety. Cyberpsychology, Behavior, and Social Networking, 16(2), 132-135. https://doi.org/10.1089/cyber.2012.0291

Bowman, L. L., Levine, L. E., Waite, B. M., \& Gendron, M. (2010). Can students really multitask? An experimental study of instant messaging while reading. Computers \& Education, 54(4), 927-931. https://doi.org/10.1016/J.COMPEDU.2009.09.024

Cain, M. S., Leonard, J. A., Gabrieli, J. D. E., \& Finn, A. S. (2016). Media multitasking in adolescence. Psychonomic Bulletin \& Review, 23(6), 1932-1941. https://doi.org/10.3758/s13423-016-1036-3

Cain, M. S., \& Mitroff, S. R. (2011). Distractor Filtering in Media Multitaskers. Perception, 40(10), 1183-1192. https://doi.org/10.1068/p7017

Cardoso-Leite, P., Kludt, R., Vignola, G., Ma, W. J., Green, C. S., \& Bavelier, D. (2016). Technology consumption and cognitive control: Contrasting action video game experience with media multitasking. Attention, Perception, \& Psychophysics, 78(1), 218-241. https://doi.org/10.3758/s13414-015-0988-0

Domingues-Montanari, S. (2017). Clinical and psychological effects of excessive screen time on children. Journal of Paediatrics and Child Health, 53(4), 333-338. https://doi.org/10.1111/jpc.13462

Edwards, K. S., \& Shin, M. (2017). Media multitasking and implicit learning. Attention, Perception, \& Psychophysics, 79(5), 1535-1549. https://doi.org/10.3758/s13414-017-13194

Gorman, T. E., \& Green, C. S. (2016). Short-term mindfulness intervention reduces the negative attentional effects associated with heavy media multitasking. Scientific Reports, 6(1), 24542. https://doi.org/10.1038/srep24542

Faul, F., Erdfelder, E., Buchner, A., \& Lang, A.-G. (2009). Statistical power analyses using G*Power 3.1: Tests for correlation and regression analyses. Behavior Research Methods, 
739

740

741

742

743

744

745

746

747

748

749

750

751

752

753

754

755

756

757

758

759

760

761

762

763

764

765

766

767

768

769

770

771

772

773

774

775

776

777

778

779

780

781

782

41, 1149-1160.

Heathcote, A., Eidels, A., Houpt, J., Colman, J., Watson, J., \& Strayer, D. (n.d.). Multi-tasking in Working Memory. Retrieved from https://psych.newcastle.edu.au/ ae273/GateKeeper/GateKee

Hooghe, M., \& Oser, J. (2015). Internet, television and social capital: the effect of 'screen time' on social capital. Information, Communication \& Society, 18(10), 1175-1199. https://doi.org/10.1080/1369118X.2015.1022568

le, A., Haller, C. S., Langer, E. J., \& Courvoisier, D. S. (2012). Mindful multitasking: The relationship between mindful flexibility and media multitasking. Computers in Human Behavior, 28(4), 1526-1532. https://doi.org/10.1016/J.CHB.2012.03.022

İmren, Mine ve Hasan Gürkan Tekman (2019). "The Relationship Between Media Multitasking, Working Memory And Sustained Attention”. Uludağ University Faculty of Arts and Sciences Journal of Social Sciences, Vol. 20, Iss. 37, p. 1075-1100.

Inzlicht, M., Shenhav, A., \& Olivola, C. Y. (2018). The Effort Paradox: Effort Is Both Costly and Valued. Trends in Cognitive Sciences, 22(4), 337-349.

https://doi.org/10.1016/J.TICS.2018.01.007

Jeong, S.-H., \& Fishbein, M. (2007). Predictors of Multitasking with Media: Media Factors and Audience Factors. Media Psychology, 10(3), 364-384. https://doi.org/10.1080/15213260701532948

Kaye, L. K., Orben, A., Ellis, D. A., Hunter, S. C., \& Houghton, S. (2020). The conceptual and methodological mayhem of "screen time." International Journal of Environmental Research and Public Health, 17(10), 3661. https://doi.org/10.3390/ijerph17103661

KONONOVA, Anastasia. Multitasking Across Borders: A Cross-National Study of Media Multitasking Behaviors, Its Antecedents, and Outcomes. International Journal of Communication, [S.I.], v. 7, p. 23, aug. 2013. ISSN 1932-8036. Available at: $<$ https://ijoc.org/index.php/ijoc/article/view/2119>.

Lascau, L., Gould, S. J. J., Cox, A. L., Karmannaya, E., \& Brumby, D. P. (2019). Monotasking or multitasking: Designing for crowdworkers' preferences. Conference on Human Factors in Computing Systems - Proceedings, 1-14. https://doi.org/10.1145/3290605.3300649

Lottridge, D. M., Rosakranse, C., Oh, C. S., Westwood, S. J., Baldoni, K. A., Mann, A. S., \& Nass, C. I. (2015). The Effects of Chronic Multitasking on Analytical Writing. Proceedings of the 33rd Annual ACM Conference on Human Factors in Computing Systems - CHI '15, 2967-2970. https://doi.org/10.1145/2702123.2702367

Lui, K. F. H., \& Wong, A. C.-N. (2012). Does media multitasking always hurt? A positive correlation between multitasking and multisensory integration. Psychonomic Bulletin \& Review, 19(4), 647-653. https://doi.org/10.3758/s13423-012-0245-7

Madore, K. P., \& Wagner, A. D. (2019). Multicosts of Multitasking. Cerebrum : The Dana Forum on Brain Science, 2019. Retrieved from http://www.ncbi.nlm.nih.gov/pubmed/32206165

Magen, H. (2017). The relations between executive functions, media multitasking and polychronicity. Computers in Human Behavior, 67, 1-9.

https://doi.org/10.1016/j.chb.2016.10.011

Mayr, U., \& Bell, T. (2006). On How to Be Unpredictable: Evidence From the Voluntary TaskSwitching Paradigm. Psychological Science, 17(9), 774-780.

https://doi.org/10.1111/j.1467-9280.2006.01781.x 
783

784

785

786

787

788

789

790

791

792

793

794

795

796

797

798

799

800

801

802

803

804

805

806

807

808

809

810

811

812

813

814

815

816

817

818

819

820

821

822

823

824

825

826

Medeiros-Ward, N., Watson, J. M., \& Strayer, D. L. (2015). On Supertaskers and the Neural Basis of Efficient Multitasking. Psychonomic Bulletin and Review, 22(3), 876-883. https://doi.org/10.3758/s13423-014-0713-3

Minear, M., Brasher, F., McCurdy, M., Lewis, J., \& Younggren, A. (2013). Working memory, fluid intelligence, and impulsiveness in heavy media multitaskers. Psychonomic Bulletin \& Review, 20(6), 1274-1281. https://doi.org/10.3758/s13423-013-0456-6

Moisala, M., Salmela, V., Hietajärvi, L., Salo, E., Carlson, S., Salonen, O., ... Alho, K. (2016). Media multitasking is associated with distractibility and increased prefrontal activity in adolescents and young adults. Neurolmage, 134, 113-121. https://doi.org/10.1016/J.NEUROIMAGE.2016.04.011

Murphy, K., \& Creux, O. (2021). Examining the association between media multitasking, and performance on working memory and inhibition tasks. Computers in Human Behavior, 114, 106532. https://doi.org/10.1016/j.chb.2020.106532

Nijboer, M., Borst, J. P., van Rijn, H., \& Taatgen, N. A. (2016). Driving and Multitasking: The Good, the Bad, and the Dangerous. Frontiers in Psychology, O(NOV), 1718. https://doi.org/10.3389/FPSYG.2016.01718

Ophir, E., Nass, C., \& Wagner, A. D. (2009). Cognitive control in media multitaskers. Proceedings of the National Academy of Sciences of the United States of America, 106(37), 15583-15587. https://doi.org/10.1073/pnas.0903620106

Orben, A., \& Przybylski, A. K. (2019). The association between adolescent well-being and digital technology use. Nature Human Behaviour, 3(2), 173-182. https://doi.org/10.1038/s41562018-0506-1

Orr, J., \& Weissman, D. (2011). Succumbing to Bottom-Up Biases on Task Choice Predicts Increased Switch Costs in the Voluntary Task Switching Paradigm . Frontiers in Psychology, Vol. 2, p. 31. Retrieved from https://www.frontiersin.org/article/10.3389/fpsyg.2011.00031

Parry, D. A., \& Le Roux, D. B. (2021). "Cognitive Control in Media Multitaskers" Ten Years On: A Meta-Analysis. Cyberpsychology, 15(2). https://doi.org/10.5817/CP2021-2-7

Patton, J. H., Stanford, M. S., \& Barratt, E. S. (1995). Factor structure of the barratt impulsiveness scale. Journal of Clinical Psychology, 51(6), 768-774. https://doi.org/10.1002/1097-4679(199511)51:6<768::AID-JCLP2270510607>3.0.CO;2-1

Pea, R., Nass, C., Meheula, L., Rance, M., Kumar, A., Bamford, H., Nass, M., Simha, A., Stillerman, B., Yang, S., Zhou, M., 2012. Media use, face-to-face communication, mediamultitasking, and social well-being among 8-to 12-year-old girls. Dev. Psychol. 48, 327. http://dx.doi.org/10.1037/a0027030.

Poposki, E. M., \& Oswald, F. L. (2010). The multitasking preference inventory: Toward an improved measure of individual differences in polychronicity. Human Performance, 23(3), 247-264. https://doi.org/10.1080/08959285.2010.487843

Ralph, B. C. W., \& Smilek, D. (2017). Individual differences in media multitasking and performance on the n-back. Attention, Perception, \& Psychophysics, 79(2), 582-592. https://doi.org/10.3758/s13414-016-1260-y

Raven, J. (1998). Manual for Raven's progressive matrices and vocabulary scales. Oxford: Oxford Psychologists.

Ralph, B. C. W., Thomson, D. R., Cheyne, J. A., \& Smilek, D. (2014). Media multitasking and

PeerJ reviewing PDF | (2021:04:60252:2:0:NEW 26 Oct 2021) 
827

828

829

830

831

832

833

834

835

836

837

838

839

840

841

842

843

844

845

846

847

848

849

850

851

852

853

854

855

856

857

858

859

860

861

862

863

864

865

866

867

868

869

870

failures of attention in everyday life. Psychological Research, 78(5), 661-669. https://doi.org/10.1007/s00426-013-0523-7

Rideout, V. J., Ulla, M. A., Foehr, G., \& Roberts, D. F. (2010). A Kaiser Family Foundation Study GENERATION M 2 Media in the Lives of 8-to 18-Year-Olds. Retrieved from https://files.eric.ed.gov/fulltext/ED527859.pdf

Robertson, I. H., Manly, T., Andrade, J., Baddeley, B. T., \& Yiend, J. (1997). 'Oops!': Performance correlates of everyday attentional failures in traumatic brain injured and normal subjects. Neuropsychologia, 35, 747-758.

Rogobete, D. A., Ionescu, T., \& Miclea, M. (2021). The Relationship Between Media Multitasking Behavior and Executive Function in Adolescence: A Replication Study. Journal of Early Adolescence, 41(5), 725-753. https://doi.org/10.1177/0272431620950478

Sanbonmatsu, D. M., Strayer, D. L., Medeiros-Ward, N., \& Watson, J. M. (2013). Who MultiTasks and Why? Multi-Tasking Ability, Perceived Multi-Tasking Ability, Impulsivity, and Sensation Seeking. PLoS ONE, 8(1), e54402. https://doi.org/10.1371/journal.pone.0054402

Schneider, D. W., \& Chun, H. (2021). Partitioning switch costs when investigating task switching in relation to media multitasking. Psychonomic Bulletin and Review, 1-8. https://doi.org/10.3758/s13423-021-01895-z

Schutten, D., Stokes, K. A., \& Arnell, K. M. (2017). I want to media multitask and I want to do it now: Individual differences in media multitasking predict delay of gratification and system-1 thinking. Cognitive Research: Principles and Implications, 2(1), 8. https://doi.org/10.1186/s41235-016-0048-x

Shin, M., Linke, A., \& Kemps, E. (2020). Moderate amounts of media multitasking are associated with optimal task performance and minimal mind wandering. Computers in Human Behavior, 111, 106422. https://doi.org/10.1016/j.chb.2020.106422

Shin, M., Webb, A., \& Kemps, E. (2019). Media multitasking, impulsivity and dual task ability. Computers in Human Behavior, 92, 160-168. https://doi.org/10.1016/J.CHB.2018.11.018

Sigman, A. (2012, November 1). Time for a view on screen time. Archives of Disease in Childhood, Vol. 97, pp. 935-942. https://doi.org/10.1136/archdischild-2012-302196

Strayer, D. L., Watson, J. M., \& Drews, F. A. (2011). Cognitive Distraction While Multitasking in the Automobile. Psychology of Learning and Motivation - Advances in Research and Theory, 54, 29-58. https://doi.org/10.1016/B978-0-12-385527-5.00002-4

Taamneh, S., Tsiamyrtzis, P., Dcosta, M., Buddharaju, P., Khatri, A., Manser, M., ... Pavlidis, I. (2017). A multimodal dataset for various forms of distracted driving. Scientific Data, 4(1), 170110. https://doi.org/10.1038/sdata.2017.110

Turner, M. L., \& Engle, R. W. (1989). Is working memory capacity task dependent? Journal of Memory and Language, 28(2), 127-154. https://doi.org/10.1016/0749-596X(89)90040-5

Wiradhany, W., \& Nieuwenstein, M. R. (2017). Cognitive control in media multitaskers: Two replication studies and a meta-Analysis. Attention, Perception, \& Psychophysics, 79(8), 2620-2641. https://doi.org/10.3758/s13414-017-1408-4

Wiradhany, W., van Vugt, M. K., \& Nieuwenstein, M. R. (2020). Media multitasking, mindwandering, and distractibility: A large-scale study. Attention, Perception, and Psychophysics, 82(3), 1112-1124. https://doi.org/10.3758/s13414-019-01842-0 
871 
Figure 1

\section{Novel Multitasking Paradigm Used}

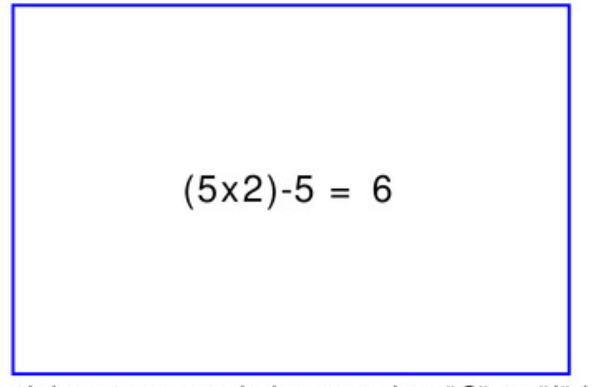

Participant responds by pressing "C" or "I" keys

" $\mathrm{C}$ " indicates the math problem is correct, while "I" indicates that it is incorrect
On 1 out of 6 trials...

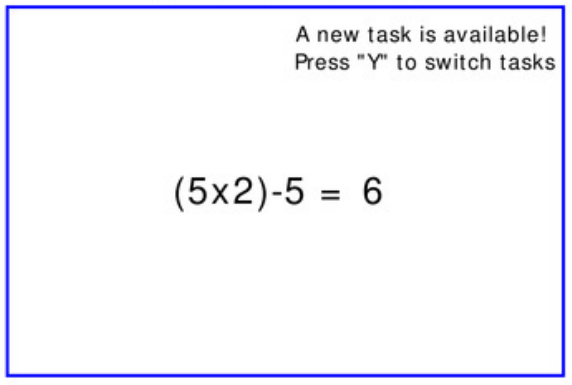

Popup prompt appears $500 \mathrm{~ms}$ after primary stimulus onset

If participant chooses to switch...

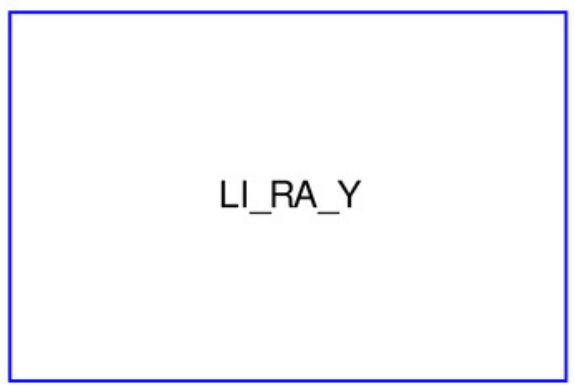

Participant responds with missing letters to complete word on screen via key press 
Figure 2

Effect of Condition on Primary Task RT

**** denotes a significance value of p. $<.0001$

Effect of Condition on Primary Task RT

Anova, $F(1.32,64.7)=24.86, p=<0.0001, \eta_{\mathrm{g}}^{2}=0.08$

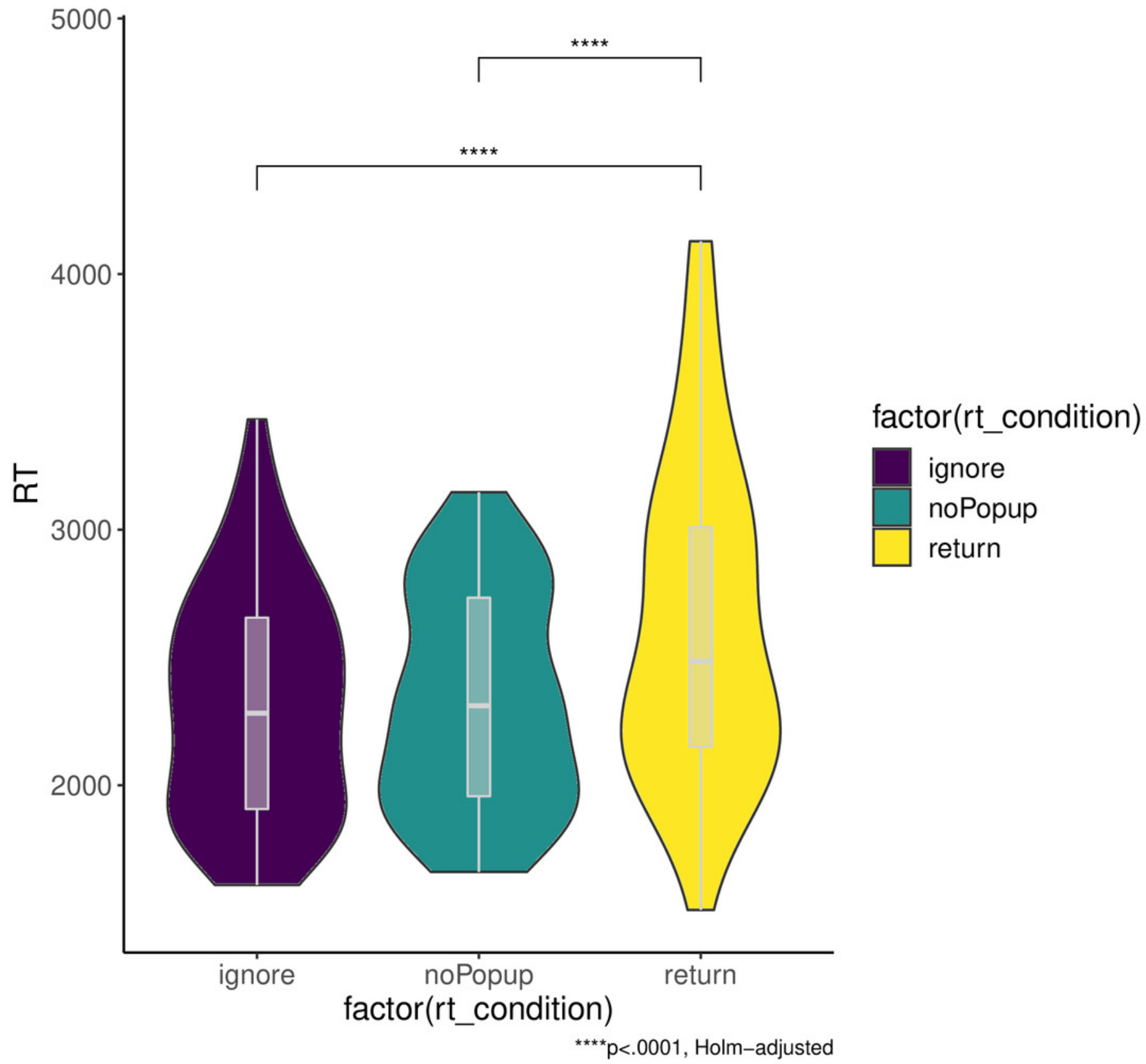


Figure 3

MMI score vs. main behavioral measures
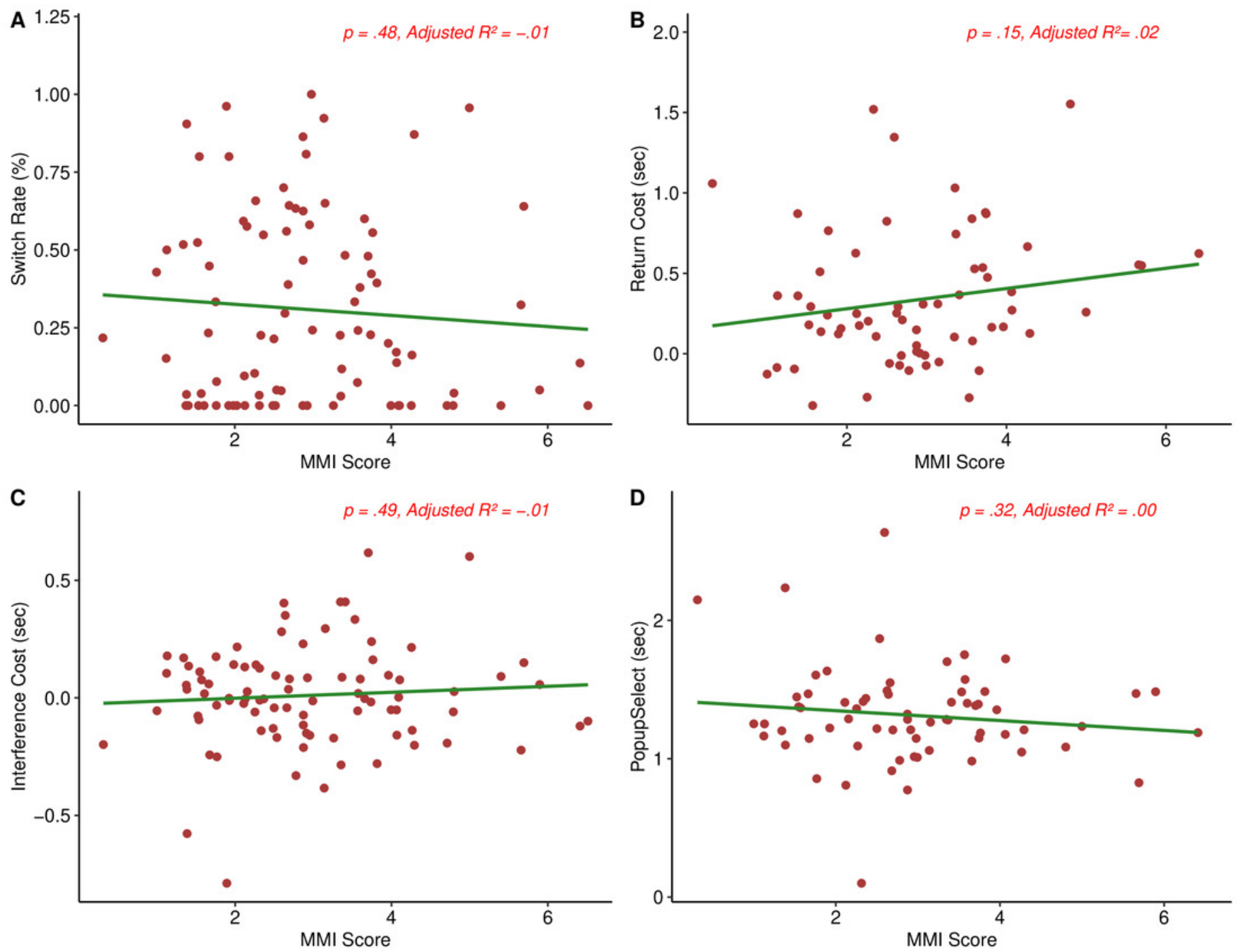
Figure 4

MMI score vs. exploratory behavioral measures
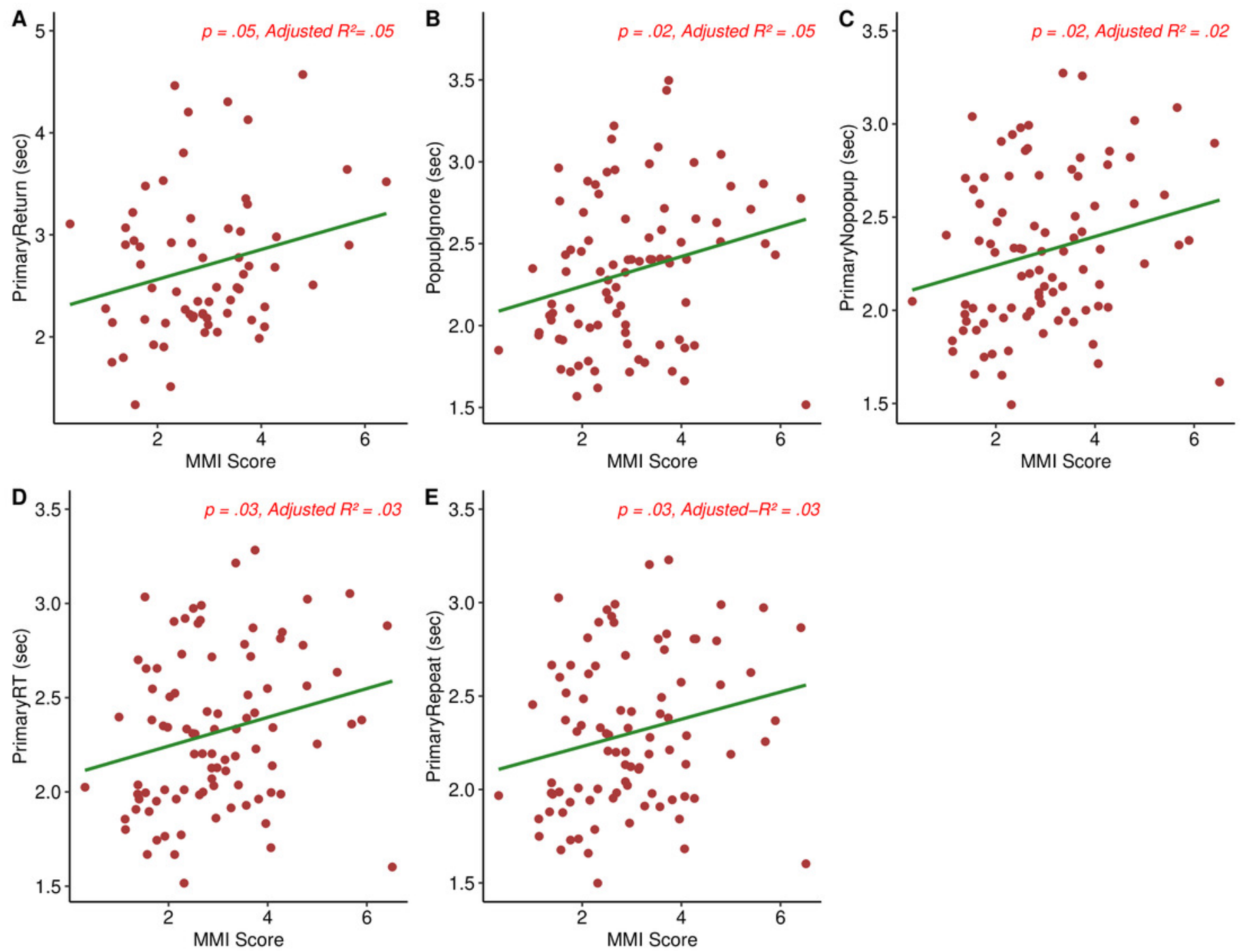


\section{Table $\mathbf{1}$ (on next page)}

Main survey descriptive statistics

Descriptive statistics for the MUQ (Media Use Questionnaire), MPI (Multitasking Preference Inventory), BIS-11 (Barratt's Impulsiveness Scale), and the three second order factors within the BIS. Note that the MUQ scores are operationalized as a Media Multitasking Index (MMI) and are presented as such in this table. 
1 Table 1. Descriptive statistics for the MUQ (Media Use Questionnaire), MPI (Multitasking

2 Preference Inventory), BIS-11 (Barratt's Impulsiveness Scale), and the three second order

3 factors within the BIS. Note that the MUQ scores are operationalized as a Media Multitasking

4 Index (MMI) and are presented as such in this table.

5

\begin{tabular}{ccccccc}
\hline & MMI Score & MPI Score & Total BIS & Attentional & Motor & Nonplanning \\
\hline Mean & 2.95 & 38.54 & 62.59 & 17.76 & 21.1 & 23.73 \\
Median & 2.82 & 37 & 61 & 17 & 20 & 23.5 \\
SD & 1.28 & 10.93 & 9.72 & 3.86 & 4.29 & 4.17 \\
\hline
\end{tabular}

6 


\section{Table 2 (on next page)}

Descriptive statistics for main behavioral measures

Descriptive statistics for the main behavioral measures analyzed. Switch rate refers to the percentage of trials in which a participant switched tasks across all relevant trials. Return cost refers to the difference in reaction time (in seconds) for primary tasks following a switch to the secondary task minus the reaction time for all other primary task trials without a popup. Interference Cost refers to the difference in reaction time (in seconds) for primary task trials with a non-selected pop-up and primary task trials without a pop-up. Finally, Popup $_{\text {select }}$ refers to the reaction time in seconds for individuals to elect to switch tasks on relevant trials 
1 Table 2. Descriptive statistics for the main behavioral measures analyzed. Switch rate 2 refers to the percentage of trials in which a participant switched tasks across all relevant trials. 3 Return cost refers to the difference in reaction time (in seconds) for primary tasks following a 4 switch to the secondary task minus the reaction time for all other primary task trials without a 5 popup. Interference Cost refers to the difference in reaction time (in seconds) for primary task 6 trials with a non-selected pop-up and primary task trials without a pop-up. Finally, Popup select 7 refers to the reaction time in seconds for individuals to elect to switch tasks on relevant trials 8 9

10

\begin{tabular}{ccccc}
\hline & $\begin{array}{c}\text { Switch } \\
\text { Rate(\%) }\end{array}$ & $\begin{array}{c}\text { Return } \\
\text { Cost }\end{array}$ & $\begin{array}{c}\text { Interference } \\
\text { Cost }\end{array}$ & Popup $_{\text {select }}$ \\
\hline Mean & 0.31 & $0.34 \mathrm{~s}$ & $0.1 \mathrm{~s}$ & $1.31 \mathrm{~s}$ \\
SD & 0.30 & $0.41 \mathrm{~s}$ & $0.22 \mathrm{~s}$ & $0.35 \mathrm{~s}$ \\
\hline
\end{tabular}

11 